16

\title{
Спектральные и рентгеновские исследования пленок оксида индия на сапфировых подложках
}

\author{
(C) А.А. Тихий ${ }^{1}$, Ю.М. Николаенко ${ }^{2}$, Ю.И. Жихарева ${ }^{3}$, И.В. Жихарев ${ }^{2}$ \\ ${ }^{1}$ Луганский национальный университет им. Тараса Шевченко, \\ 91011 Луганск, Украина \\ ${ }^{2}$ Донецкий фозико-технический институт им. А.А. Галкина, \\ 83114 Донецк, Украина \\ ${ }^{3}$ Киевский национальный университет им. Тараса Шевченко, \\ 01601 Киев, Украина \\ e-mail: ea0000ffff@mail.ru
}

Поступила в редакцию 26.04.2020 г.

В окончательной редакции 07.06.2020 г.

Принята к публикации 16.06.2020 г.

\begin{abstract}
Представлены результаты исследования спектров оптического пропускания и рентгеновской дифракции тонких пленок $\mathrm{In}_{2} \mathrm{O}_{3}$, полученных методом DC-магнетронного распыления на подложках $\mathrm{Al}_{2} \mathrm{O}_{3}(012)$. В дифрактограммах присутствует рефлекс, соответствующий плоскости (222) кубического $\operatorname{In}_{2} \mathrm{O}_{3}$. Его положение смещается с 30.3 к $30.6^{\circ}$ при уменьшении толщины пленки. Полуширина этого рефлекса убывает с уменьшением времени напыления, что может указывать на увеличение размера зерен материала пленки. Согласно измерениям оптического пропускания, на границе раздела между пленкой и подложкой было установлено наличие переходного слоя с шириной запрещенной зоны $1.39 \mathrm{eV}$ и толщиной около $40 \mathrm{~nm}$. Свойства этого слоя не зависят от времени напыления.
\end{abstract}

Ключевые слова: оксид индия, тонкие пленки, оптическое пропускание, рентгеноструктурный анализ.

DOI: $10.21883 /$ OS.2020.10.50029.138-20

\section{1. Введение}

$\mathrm{In}_{2} \mathrm{O}_{3}$ является достаточно известным и хорошо изученным полупроводником. Тем не менее, благодаря уникальному сочетанию в них свойств высокой оптической прозрачности $(>80 \%)$ и высокой электропроводности, которая к тому же очень чувствительна к составу окружающей атмосферы интерес исследователей к пленкам этого материала не ослабевает и по сей день. Легирование $\mathrm{In}_{2} \mathrm{O}_{3}$ различными примесями позволяет широко варьировать свойства пленок - электропроводность, чувствительность к различным газам и т.д. [1-3]. Благодаря своим свойствам пленки на основе $\operatorname{In}_{2} \mathrm{O}_{3}$ уже нашли широкое применение в качестве прозрачных проводников, а также являются перспективным материалом для создания газовых датчиков. Для обеспечения высокой чувствительности последних, важно наличие развитой поверхности, на которой происходит обратимое связывание аналита. Наличие значительной шероховатости на поверхности пленок $\mathrm{In}_{2} \mathrm{O}_{3}$, которая естественным образом образуется в процессе их роста, обеспечивает большую площадь контакта с окружающей средой. Поликристаллическая структура пленок и присутствие в них механических напряжений также увеличивают коэффициенты диффузии [4] и, соответственно, увеличивают чувствительность сенсоров на их основе.

Исследование оптического пропускания тонких пленок позволяет изучать не только структуру энергетиче- ских уровней материала, но и определять количество, а также толщину слоев многослойных покрытий.

Используемый нами метод магнетронного распыления является масштабируемым и обеспечивает хорошую воспроизводимость параметров пленок.

\section{2. Эксперимент}

Исследованные пленки получены на подложках $\mathrm{Al}_{2} \mathrm{O}_{3}$ (012) методом dс-магнетронного распыления мишени $\mathrm{In}_{2} \mathrm{O}_{3}$ [5]. Напыление производилось в течение 15, $35,45,60,120$ и $180 \mathrm{~min}$ при токе $50 \mathrm{~mA}$ и напряжении $300 \mathrm{~V}$. Температура подложек в процессе напыления составляла $600^{\circ} \mathrm{C}$.

Пленки исследованы рентгеновским и спектрофотометрическим методами. Рентгеновские измерения проводились с использованием излучения $\mathrm{Cu}_{\alpha}$ на дифрактометре ДРОН-3 оснащенном модернизированной системой автоматического управления и регистрации данных. Измерения оптического пропускания выполнены на спектрофотометре Shimadzu UV-2450 в диапазоне длин волн от 200 до $1000 \mathrm{~nm}$.

\section{3. Результаты и обсуждение}

Полученные дифрактограммы пленок содержат рефлекс, который смещается от 30.3 до $30.6^{\circ}$ в зависимости от времени осаждения (рис. 1). Его положение 


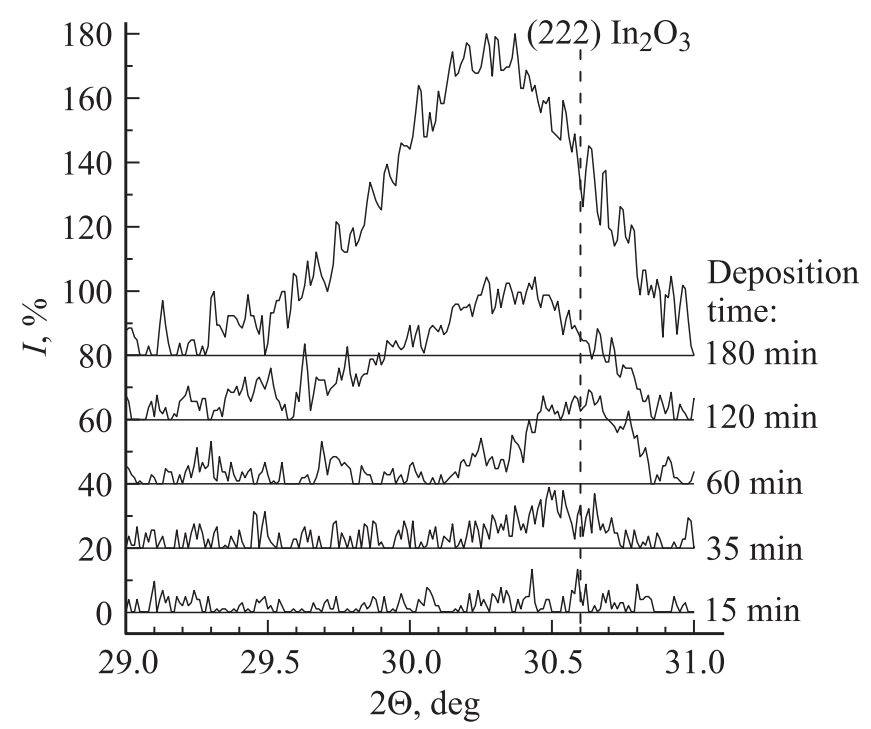

Рис. 1. Дифрактограммы исследованных пленок.

близко к положению рефлекса, соответствующего плоскости (222) кубической решетки $\operatorname{In}_{2} \mathrm{O}_{3}$ (пространственная группа $I a \overline{3})$, согласно данным [6]. Это, а также закономерное уменьшение интенсивности наблюдаемого рефлекса с уменьшением времени осаждения пленки позволяет связать его с плоскостью (222) материала пленки. Полуширина данного рефлекса уменьшается с уменьшением времени осаждения. Это указывает на то, что средний размер зерен плёнок с малым временем напыления больше, чем плёнок с большим временем напыления. Подобную зависимость размеров зерен от времени напыления, по результатам рентгеноструктурных измерений, на аналогичных плёнках наблюдали также авторы [7].

Поворот образцов в плоскости их поверхности вызывает резкое изменение интенсивности рефлексов подложки $\mathrm{Al}_{2} \mathrm{O}_{3}$ от нуля до максимума, что указывает на некоторую разориентацию плоскости среза с плоскостью (012) $\mathrm{Al}_{2} \mathrm{O}_{3}$. Однако такой поворот практически не влияет на интенсивность рефлекса вблизи $306^{\circ}$, что подтверждает его принадлежность к материалу пленки и говорит о поликристалличности полученных плёнок.

Результаты измерений оптического пропускания демонстрируют его аномальное уменьшение с уменьшением длины волны. Кроме того, на кривых оптического пропускания пленок с большим временем напыления интерференционная картина наблюдается даже в области низкого пропускания. Для объяснения этих результатов была построена модель исследованных пленок (рис. 2). Согласно этой модели, исследуемые пленки состоят из трех слоев. Оптические свойства среднего слоя соответствуют кубической модификации $\mathrm{In}_{2} \mathrm{O}_{3}$ (пространственная группа $I a \overline{3})$ согласно [7]. Поверхность пленок предполагается шероховатой и смоделирована как однородный слой с оптическими свойствами, рассчитанными на основе диэлектрической проницаемости кубической модификации $\operatorname{In}_{2} \mathrm{O}_{3}$ и коэффициента заполнения 0.5 , в соответствии с уравнением Клаузиуса-Моссотти. Это является обычной практикой при моделировании шероховатой поверхности подобных пленок $[9,10]$.

Однако наличие только указанных слоев не полностью объясняет наблюдаемые спектры оптического пропускания. Для их полного объяснения в модель вводится третий слой, расположенный между пленкой и подложкой. Из экспериментальных данных очевидно, что он имеет высокий коэффициент экстинкции. Наилучшим описанием спектральной зависимости коэффициента экстинкции этого слоя является закон фундаментального поглощения в полупроводнике с шириной запрещенной зоны $E_{g}=1.39 \mathrm{eV}$ для прямых переходов:

$$
k(h v)=A\left(h v-E_{g}\right)^{1 / 2},
$$

где $h v-$ энергия фотона, $A-$ постоянная, которая не зависит от энергии фотона. Поскольку значение коэффициента экстинкции данного слоя велико (мнимая часть диэлектрической проницаемости намного больше действительной), вклад показателя преломления в коэффициенты отражения и пропускания этого слоя мал. Следовательно, сложно однозначно определить его значение по результатам измерений. Поэтому в качестве показателя преломления этого слоя можно использовать любое небольшое число, однако, исходя из предположения о некотором сходстве его состава с составом материала пленки, ему была присвоена та же спектральная зависимость показателя преломления, что и для среднего слоя модели пленки. Кроме того, из-за высокого поглощения трудно оценить толщину $d_{3}$ этого слоя. Значение $A$ принималось равным единице, поскольку его вклад в коэффициенты отражения и пропускания этого слоя нельзя однозначно отделить от вклада $d_{3}$.

Air

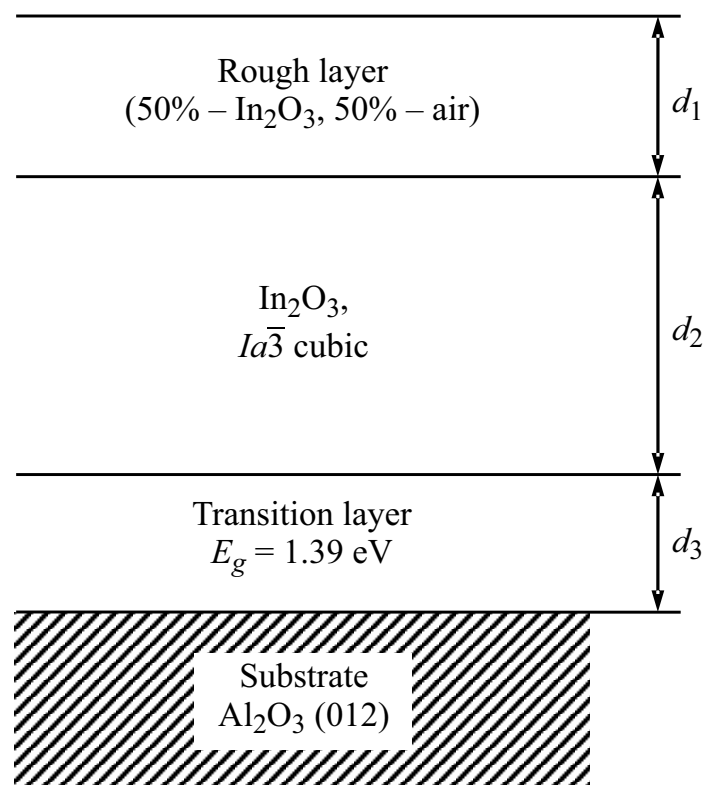

Рис. 2. Модель исследованных пленок. 
Зависимость толщины слоев модели исследованных пленок от времени напыления

\begin{tabular}{c|c|c|c}
\hline Время напыления, $\min$ & $d_{1}, \mathrm{~nm}$ & $d_{2}, \mathrm{~nm}$ & $d_{3}, \mathrm{~nm}$ \\
\hline 15 & 60 & 5 & 32 \\
35 & 60 & 25 & 40 \\
60 & 40 & 60 & 40 \\
120 & 60 & 105 & 28 \\
180 & 70 & 290 & 32
\end{tabular}

Вклад отражения от подложки в этой системе не играет существенной роли, тем не менее, дисперсия его показателя преломления была учтена.

В целом пропускание для выбранной модели исследуемых пленок рассчитывалось по точным формулам с использованием матрицы рассеяния для системы плоскопараллельных однородных изотропных слоев [10]. Также учитывалась немонохроматичность зондирующего излучения путем усреднения коэффициента пропускания в диапазоне длин волн, соответствующем использованной спектральной ширине щели спектрофотометpa $-5 \mathrm{~nm}$.

Толщины всех слоев подбирались вручную, до достижения соответствия рассчитанных спектров пропускания экспериментальным (таблица).

Сравнение спектров оптического пропускания, рассчитанных в рамках предлагаемой модели, с результатами измерений дает хорошее согласие во всем исследованном диапазоне длин волн, за исключением области 300-400 nm (рис. 3). Согласно данным [7], в этой области начинается резкое увеличение показателя преломления $\mathrm{In}_{2} \mathrm{O}_{3}$, что связано с началом фундаментального поглощения. По-видимому, ширина запрещенной зоны $\mathrm{In}_{2} \mathrm{O}_{3}$ в исследованных нами пленках несколько больше, чем в пленках, исследованных авторами [7]. Это неудивительно, поскольку для этого материала характерен некоторый разброс значений ширины запрещенной зоне в зависимости от условий получения [11].

Образование переходного слоя на границе с подложкой, по-видимому, не требует большого нарушения стехиометрии пленки, так как это, возможно, связано с размытием запрещенной зоны из-за большого количества дефектов в кристаллической структуре, а также образования примесных уровней внутри запрещенной зоны. Толщина этого слоя практически не зависит от времени напыления, и, следовательно, его появление полностью обусловлено влиянием поверхности подложки.

Толщина шероховатого слоя на поверхности пленки также практически не зависит от времени напыления и определяется в основном режимом напыления.

Отсутствие корреляции толщин переходных слоев с размерами зёрен, возможно, объясняется тем, что результаты рентгеновских измерений позволяют судить об их средней величине, но при таких толщинах покрытия ничего не говорят о распределении размеров зерен в объеме пленки, ведь глубина проникновения рентгенов-

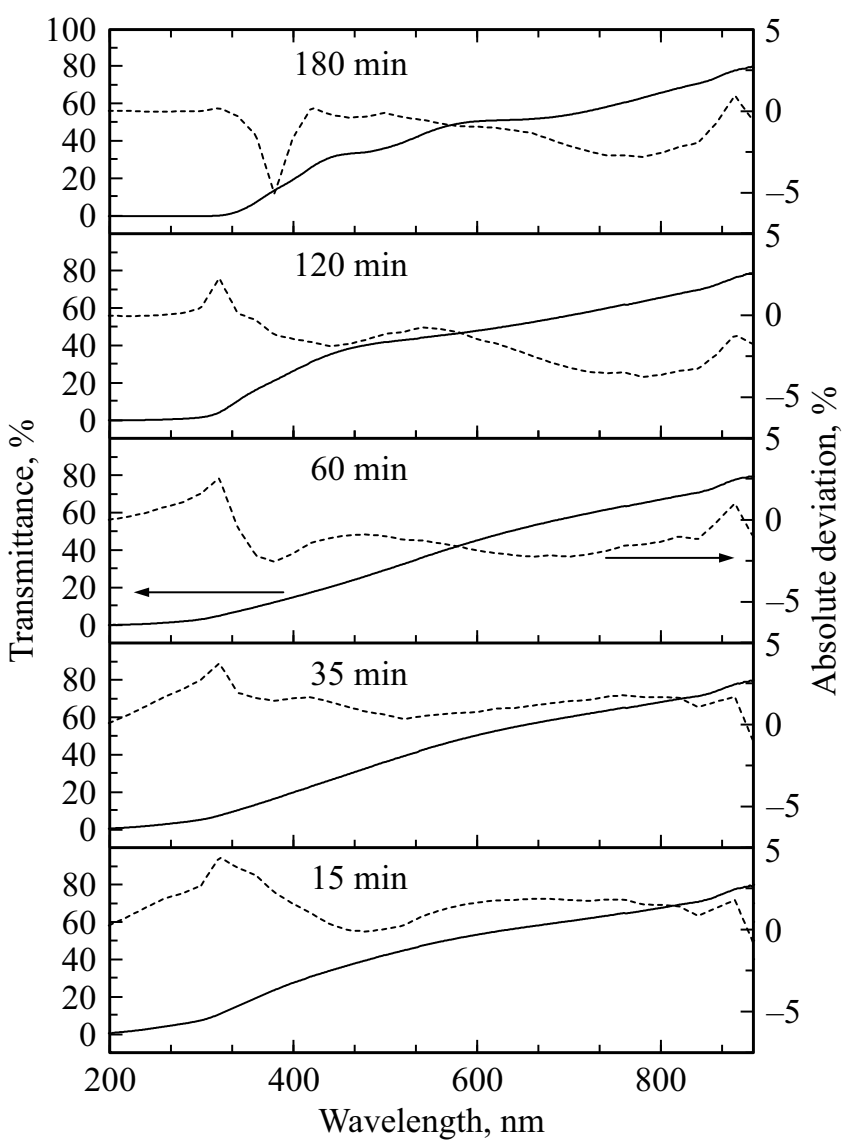

Рис. 3. Сравнение расчетных (штриховая линия) и измеренных оптических спектров пропускания (сплошная линия) исследованных пленок. Время напыления показано на графиках.

ского излучения составляет порядка нескольких микрометров. То есть вклад различных слоёв данных плёнок картину дифракции рентгеновских лучей практически не зависит от того, на каком расстоянии от поверхности плёнки они находятся.

Отсутствие корреляции толщин переходных слоев с размерами зёрен, возможно, объясняется тем, что результаты рентгеновских измерений позволяют судить об их средней величине, но при таких толщинах покрытия ничего не говорят о распределении размеров зерен в объеме пленки, ведь глубина проникновения рентгеновского излучения составляет порядка нескольких микрометров. То есть вклад различных слоёв данных плёнок картину дифракции рентгеновских лучей практически не зависит от того, на каком расстоянии от поверхности плёнки они находятся.

Отсутствие корреляции толщин переходных слоев с размерами зёрен, возможно, объясняется тем, что результаты рентгеновских измерений позволяют судить об их средней величине, но при таких толщинах покрытия ничего не говорят о распределении размеров зерен в объеме пленки, ведь глубина проникновения рентгеновского излучения составляет порядка нескольких микрометров. То есть вклад различных слоёв данных плёнок 
картину дифракции рентгеновских лучей практически не зависит от того, на каком расстоянии от поверхности плёнки они находятся.

\section{4. Выводы}

Предложенная модель исследованных пленок позволяет описать их оптические спектры пропускания. Структура и свойства основного объема пленок, в целом, соответствуют структуре и свойствам таких пленок, представленным в литературе. Результаты рентгеноструктурных исследований указывают на увеличение размера зерна $\mathrm{In}_{2} \mathrm{O}_{3}$ при уменьшении толщины пленки. Сдвиг рефлекса, соответствующего плоскости (222), с увеличением времени напыления, а также наличие переходного слоя с шириной запрещенной зоны $1.39 \mathrm{eV}$ на границе пленка-подложка обусловлены влиянием подложки на структуру материала пленки.

\section{Конфликт интересов}

Авторы заявляют, что у них нет конфликта интересов.

\section{Список литературы}

[1] Korotcenkov G., Brinzari V., Cho B.K. // J. of Sensors. 2016. V. 2016. Article ID 3816094. 31 p. doi 10.1155/2016/3816094

[2] Abbasi M., Rozati S.M. // J. of Electronic Materials. 2016. V. 45 P. 2855. doi 10.1007/s11664-015-4315-2

[3] Kadhim M.A., Ramadhan A.A., Al-Gburi M.O.S., Habi G.J., Hentawe N.J. // Karbala International J. of Modern Science. 2020. V. 6. Iss. 1. Article 12. doi 10.33640/2405-609X.1403

[4] Nikolaenko Yu.M., Artemov A.N., Medvedev Yu.V., Efros N.B., Zhikharev I.V., Reshidova I.Yu., Tikhii A.A., KaraMurza S.V. // J. Phys. D: Appl. Phys. 2016. V. 49. P. 375302. doi 10.1088/0022-3727/49/37/375302

[5] Николаенко Ю.М., Мухин А.Б., Чайка В.А., Бурховещкий В.В. // ЖТФ. 2010. Т. 80. С. 115; Nikolaenko Yu.M., Mukhin A.B., Chaika V.A., Burkhovetskii V.V. // Tech. Phys. 2010. V. 55 P. 1189. doi 10.1134/S1063784210080189

[6] Swanson H.E., Gilfrich N.T., Ugrinic G.M. // Circular of the Bureau of Standards no. 539. V. 5: standard x-ray diffraction powder patterns, National Bureau of Standards (US). 1955. P. 26.

[7] Ghorannevis Z., Akbarnejad E., Ghoranneviss M. // J. Theor Appl. Phys. 2015. V. 9. P. 285. doi 10.1007/s40094-015-01873

[8] Lehmann D., Seidel F., Zahn D.R.T. // SpringerPlus. 2014. V. 3. P. 82. doi 10.1186/2193-1801-3-82

[9] Tikhii A.A., Nikolaenko Yu.M., Kornievets A.S., Svyrydova K.A., Zhikhareva Yu.I., Zhikharev I.V. // J. Appl. Spectrosc. 2019. V. 86. P. 1053. doi 10.1007/s10812-02000939-x

[10] Tompkins H.G., Irene E.A. (Eds), Handbook of Ellipsometry. USA: William Andrew Publishing, 2005. 891 p.

[11] Jarzebski Z.M. // Phys. Stat. Sol. (a). 1982. V. 71 P. 13. doi 10.1002/pssa.2210710102 